\title{
ANÁLISE DA ESTABILIDADE DA REDE GEODÉSICA DE MONITORAMENTO DA UHE SALTO CAXIAS A PARTIR DE DADOS GPS
}

\author{
Stability analysis of geodetic network of Salto Caxias Hydroelectric Power Station \\ from GPS data \\ FELIPE MENDES SUCI ${ }^{1}$ \\ PEDRO LUIS FAGGION ${ }^{2}$ \\ 'Petróleo Brasileiro S/A - Petrobras \\ Unidade de Operações de Exploração e Produção do Rio Grande do Norte e Ceará \\ BR-304, Km 46, CEP 59.633-900, Mossoró, RN \\ ${ }^{2}$ Universidade Federal do Paraná - UFPR \\ Programa de Pós-Graduação em Ciências Geodésicas \\ Setor de Ciências da Terra - Departamento de Geomática \\ Caixa Postal 19001, CEP 81.531-990 Curitiba, PR \\ \{felipesuci@petrobras.com.br; faggion@ufpr.br\}
}

\begin{abstract}
RESUMO
O presente artigo busca analisar a estabilidade da rede geodésica que dá suporte ao monitoramento da Usina Hidrelétrica Salto Caxias. Tal rede é composta por cinco pilares de centragem forçada, localizados à jusante da barragem, além de um pino de crista, situado sobre uma das comportas da mesma. Essa análise foi realizada a partir da coleta de dados GPS, os quais foram processados em software apropriado para tal fim. Decidiu-se tomar o pilar P1 como referência e suas coordenadas foram determinadas de forma relativa (utilizando-se o software Leica Geo Office v.7.0) em relação a quatro estações de monitoramento contínuo, sendo três pertencentes à RBMC (PRMA, PRGU e SCCH) e uma pertencente à Itaipu Binacional (ITAI). As coordenadas dessas bases foram transformadas e atualizadas ao ITRFyy e época (com a Transformação Generalizada de Helmert) correspondentes às efemérides precisas utilizadas e à época de rastreio. As coordenadas dos outros pontos da rede foram determinadas em relação ao P1, a partir do posicionamento relativo. Para se detectar observações com erros grosseiros, foi aplicado o teste Data Snooping. Tais coordenadas foram transformadas de um sistema geocêntrico para um sistema geodésico local, com a finalidade de melhor avaliar os deslocamentos, que por sua
\end{abstract}


vez, foram validados a partir do Teste de Congruência Global. Por fím, com o objetivo de avaliar as distâncias entre os pilares, obtidas com dados GPS, as mesmas foram comparadas com as distâncias obtidas com a ET Leica TC2003. Os resultados demonstraram que os deslocamentos detectados a partir dos dados GPS não foram compatíveis com os resultados obtidos com a técnica de trilateração.

Palavras-Chave: Monitoramento; Redes Geodésicas; Teste de Congruência Global.

\section{ABSTRACT}

This paper aims at presenting the analysis of the stability of the geodetic network that supports the monitoring of Salto Caxias Hydroelectric Plant. This network consists of five pillars equipped with devices of forced centering, which are located downstream of the dam, and a pin fixed in the crest, located in one of the gates of the dam. The analysis was performed from the GPS data collection and the processing by using appropriate software. We decided to take pillar P1 as reference and its coordinates were determined by relative form (using the Leica Geo Office software v.7.0) four continuous monitoring stations, three belonging to RBMC (PRMA, PRGU and SCCH) and one to Itaipu (ITAI). The coordinates of these bases were transformed and updated to ITRFyy and reference epoch (from Generalized Helmert Transformation) corresponding to the precise ephemeris used and the epoch of data collection. The coordinates of other points of the network were determined in relation to $\mathrm{P} 1$, using the relative positioning. To detect observations with blunders, we applied the Data Snooping procedure. The coordinates were transformed from a geocentric to a local geodetic system, in order to better evaluate the displacements, which in turn were validated from the Global Congruency Test. Finally, in order to evaluate the distance between the pillars obtained by GPS data they were compared with the distances obtained with the Total Station Leica TC2003. The results showed that the displacements obtained from GPS data were not consistent with the results obtained from trilateration technique.

Keywords: Monitoring; Geodetic Networks; Global Congruency Test.

\section{INTRODUÇÃO}

O estudo sobre a estabilidade de uma rede geodésica de monitoramento é de extrema importância na análise de deslocamentos que possam estar ocorrendo na obra de engenharia monitorada.

Pontes, pórticos, portos, edifícios e barragens, são alguns exemplos de obras de engenharia que devem ser monitoradas. No presente estudo, o enfoque será dado ao monitoramento geodésico de barragens, uma vez que a rede geodésica em análise localiza-se na região da UHE Salto Caxias, localizada no sudoeste paranaense, entre os municípios de Capitão Leônidas Marques e Nova Prata do Iguaçu.

Barragens são monitoradas a partir de métodos geodésicos e geotécnicos e ambos se tornam complementares, visto que o primeiro fornece informações de forma global (em relação a um sistema de referência pré-definido) e o segundo 
oferece maior precisão, mas sem a vantagem de ter um sistema de referência fora da estrutura. $\mathrm{O}$ interesse do presente trabalho recai sobre os métodos geodésicos e de acordo com US ARMY (2002) os mesmos podem ser baseados em técnicas terrestres (ex: triangulateração e nivelamento geométrico) ou espaciais (ex: GPS).

Dados geotécnicos e geodésicos podem ser analisados de forma solidária, onde as observações obtidas pelos métodos, quando combinadas, fornecem informações relacionadas à estabilidade da estrutura monitorada. A coleta, processamento e análise das observações podem ser realizados de forma automatizada, diminuindo o tempo de resposta e a probabilidade de erros por parte do operador (DUFFY et. al., 2001; KÄLBER et. al., 2000).

No Brasil, tem-se destacado as pesquisas desenvolvidas por diferentes centros como a Universidade Federal do Paraná (UFPR) e Universidade de São Paulo (USP). Autores como Fazan (2010) e Ribeiro (2008) descrevem metodologias utilizadas na análise, validação e representação de deslocamentos da rede geodésica da barragem de Itaipu a partir de dados GPS. Por sua vez, a barragem da UHE Salto Caxias vem sendo analisada desde 1998 (GRANEMANN, 2005; NADAL, 2008; NEVES, 2008)), tomando como referência a rede geodésica ali instalada, composta por 5 pilares de centragem forçada à jusante da barragem e um pino localizado sobre a crista da mesma a partir de técnicas geodésicas locais (triangulação, trilateração, poligonação geodésica e nivelamento geométrico).

Estudos sobre a estabilidade de tal rede geodésica, entretanto, não têm sido realizados em relação a um sistema geodésico externo à área de influência da barragem, tornando-se assim, objeto de pesquisa do presente trabalho. Na Figura 1 é possível visualizar a disposição da rede geodésica estudada e os vetores formados entre os pontos de interesse, além de ser possível verificar a proximidade dos mesmos em relação à barragem.

Figura 1 - Rede geodésica da UHE Salto Caxias. Fonte: USINA Hidrelétrica Salto Caxias (2003).

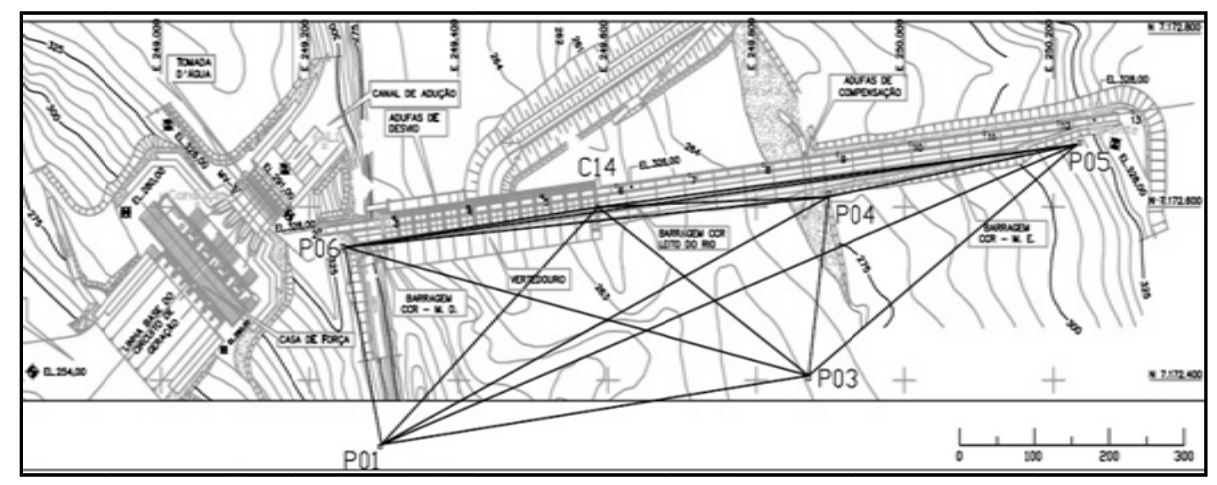


A coleta de dados GPS dá início ao processo de análise de estabilidade dos pilares da rede geodésica da UHE Salto Caxias. Os mesmos são então processados e ajustados no aplicativo Leica Geo Office v.7.0.. No processamento foram utilizadas efemérides precisas IGS, sendo essas referenciadas ao ITRF05 e ITRF08, por esse motivo, torna-se necessária a aplicação da Transformação Generalizada de Helmert para se adequar os sistemas de referência, já que as efemérides transmitidas (dados rastreados) estão referenciadas ao WGS84, que aqui será tomado como compatível ao ITRF00.

A atualização das coordenadas também tem o objetivo de compatibilizá-las temporalmente, ou seja, a comparação entre as coordenadas será realizada levandose em consideração o movimento da placa litosférica, impedindo assim que o mesmo seja interpretado de forma errônea como deslocamento do pilar.

Para auxiliar a análise dos dados, foram plotados gráficos que mostram o comportamento das componentes das coordenadas dos pilares durante as campanhas.

A última análise baseou-se em comparar as distâncias obtidas com os dados GPS e com a Estação Total Leica TC2003, com precisão nominal linear de $\pm(1 \mathrm{~mm}$ $+1 \mathrm{ppm})$.

\section{CARACTERÍSTICAS DA ÁREA DE ESTUDO}

A UHE Salto Caxias (Usina Governador José Richa) localiza-se entre as cidades de Capitão Leônidas Marques e Nova Prata do Iguaçu, aproximadamente a $600 \mathrm{~km}$ de Curitiba.

De acordo com COPEL (2008), a UHE Salto Caxias tem capacidade instalada de $1240 \mathrm{MW}$ com volume total acumulado de 3,5 bi $\mathrm{m}^{3}$ de água. A barragem, em seu ponto mais alto tem $67 \mathrm{~m}$ de altura (da fundação à crista) e $1083 \mathrm{~m}$ de comprimento e foram utilizados $913 \mathrm{mil} \mathrm{m}^{3}$ de concreto compactado a rolo (CCR) para a sua construção, tornando-a assim a $8^{\mathrm{a}}$ maior barragem de CCR do mundo e a maior do Brasil. A vazão em tempos de cheia chega a $49,6 \mathrm{mil} \mathrm{m}^{3} / \mathrm{s}$, sendo essa garantida por 14 comportas, cada uma com 16,5 m de largura e $20 \mathrm{~m}$ de comprimento.

Em relação à instrumentação da barragem, de acordo com Junior e Soares (2006), 87\% do instrumental é automatizado, com tomada e acesso aos dados de forma remota e os outros $13 \%$ são compostos de instrumentos não automatizados.

A instrumentação total, composta por 191 equipamentos é descrita a seguir (JUNIOR e SOARES, 2006):

- Automatizados:

o 64 Termômetros;

o 28 Medidores de Junta;

o 23 Piezômetros de Maciço;

o 41 Piezômetros de Fundação;

o 08 Medidores de Vazão;

o 03 Pêndulos; 
- Não-automatizados:

o 06 Extensores Múltiplos;

o 05 Medidores Triortogonais;

o 13 Marcos Topográficos (instrumentação geodésica).

Além de 977 Drenos de Cortina, que mesmo sendo considerados instrumentos de drenagem, têm os seus dados analisados juntamente com o restante da instrumentação. A instrumentação geodésica conta ainda, conforme já mencionado, com 6 pilares dotados de dispositivos de centragem forçada à jusante da barragem, além de Marcos Geodésicos Internos (MGI's) e Externos (MGE's).

\section{METODOLOGIA}

\subsection{Coleta dos dados GPS}

$\mathrm{Na}$ Tabela 1 são apresentadas as épocas em que ocorreram as coletas dos dados GPS utilizados no presente trabalho.

Tabela 1 - Épocas Utilizadas.

\begin{tabular}{|c|c|c|c|}
\hline \multicolumn{2}{|c|}{ Campanha } & \multirow{2}{*}{ Semana GPS } & \multirow{2}{*}{ Dias do ano } \\
\hline Mês & Ano & & \\
\hline Fev & 2009 & 1518 & 42,43 \\
\hline Jun & \multirow{2}{*}{2010} & 1588 & $165,166,167,168$ \\
\hline Out & & 1607 & $298,299,300,301$ \\
\hline Mai & \multirow{2}{*}{2011} & 1637 & $143,144,145,146$ \\
\hline Out & & 1658 & $290,291,292,293$ \\
\hline
\end{tabular}

Para a coleta dos dados foram utilizados seis receptores GPS e os mesmos são descritos na Tabela 2, juntamente com suas características. Ressalta-se que todos os aparelhos são de dupla frequência (L1/L2).

Tabela 2 - Aparelhos Utilizados e Suas Precisões Nominais.

\begin{tabular}{c|c|c|c}
\hline \multirow{2}{*}{ Modelo (Qtde.) } & \multirow{2}{*}{ Antena } & \multicolumn{2}{|c}{ Precisão Nominal } \\
\cline { 3 - 4 } & & Horizontal & Vertical \\
\hline GX1230 Leica (1) & AX1202 GG & $\pm(5 \mathrm{~mm}+0,5 \mathrm{ppm})$ & $\pm(10 \mathrm{~mm}+0,5 \mathrm{ppm})$ \\
\hline GX1230 Leica (1) & AX1230 & $\pm(5 \mathrm{~mm}+0,5 \mathrm{ppm})$ & $\pm(10 \mathrm{~mm}+0,5 \mathrm{ppm})$ \\
\hline 900CS Leica (2) & ATX900CS & $\pm(5 \mathrm{~mm}+0,5 \mathrm{ppm})$ & $\pm(10 \mathrm{~mm}+0,5 \mathrm{ppm})$ \\
\hline Hiper Lite Topcon (2) & - & $\pm(3 \mathrm{~mm}+0,5 \mathrm{ppm})$ & $\pm(5 \mathrm{~mm}+0,5 \mathrm{ppm})$ \\
\hline
\end{tabular}

Alguns parâmetros foram adotados para a coleta dos dados, a saber:

- Máscara de elevação: $10^{\circ}$;

- Taxa de gravação: 5 segundos; 
- Tempo de rastreio: $>6$ horas, e;

- Seção única (em cada um dos dias).

\subsection{Atualização de coordenadas - VEMOS2009}

Em aplicações geodésicas onde alta precisão é requerida, é necessário que os movimentos das placas litosféricas sejam considerados. De acordo com Seeber (1993), tais movimentos são descritos por modelos teóricos de velocidade de placas.

US ARMY (2002, pg. 2-2) sugere que alguns limites mínimos de precisão requerida sejam seguidos no monitoramento de estruturas de concreto, a saber: \pm 5 $10 \mathrm{~mm}$ no monitoramento de movimentos de baixa frequência; $\pm 0,2 \mathrm{~mm}$ no monitoramento de movimentos de alta frequência e em fissuras, e; $\pm 2 \mathrm{~mm}$ em movimentos verticais (subsidência, soerguimento, recalque).

A atualização das coordenadas dos pontos de interesse é realizada conhecendo-se as coordenadas de origem (ou rastreio) de tais pontos, a época de origem, a época de destino, além do vetor de velocidades, sendo esse fornecido pelo modelo teórico adotado.

O modelo oficial adotado pelo SIRGAS e pelo Instituto Brasileiro de Geografia e Estatística (IBGE) é o Modelo de Velocidades para a América do Sul e Caribe (VEMOS2009), apresentado em Drewes e Heidbach (2009). As velocidades dos pontos de interesse são determinadas a partir do aplicativo VMS2009, disponível no sítio do SIRGAS (http://www.sirgas.org/index.php?id=54). A saber, as velocidades médias para as coordenadas cartesianas geocêntricas (X, Y, Z), fornecidas para a região da UHE Salto Caxias são: $1,6 \mathrm{~mm} / \mathrm{ano}(\mathrm{Vx})$; -5,5 mm/ano (Vy) e; 11,3 mm/ano (Vz).

\subsection{Transformação Generalizada de Helmert}

No processamento dos dados GPS foram utilizadas efemérides precisas IGS. As mesmas estão referenciadas a diferentes realizações do International Terrestrial Reference Frame (ITRFyy), onde yy significa o ano da realização em questão. No processamento relativo, foram utilizadas bases da Rede Brasileira de Monitoramento Contínuo (RBMC), os dados dessas bases estão no formato RINEX e referenciados ao SIRGAS2000 (compatível com o ITRF00), porém, as efemérides precisas utilizadas são referenciadas a outras versões do ITRF (05 e 08), sendo necessária a transformação entre as mesmas.

Essa operação é realizada pela Transformação Generalizada de Helmert (TGH), na qual são utilizados quatorze parâmetros (os sete parâmetros de similaridade e suas variações temporais). De acordo com Leick (2004) e Monico (2008), a TGH é dada por:

$$
\begin{gathered}
\vec{X}_{B(t)}=\vec{T}+(1+s)[\varepsilon+I]\left[\vec{X}_{A\left(t_{0}\right)}+\vec{V}_{A\left(t_{0}\right)}\left(t-t_{0}\right)\right] \\
+\left[\dot{\vec{T}}+[(1+s) \dot{\varepsilon}+\dot{s}[\varepsilon+I]] \vec{X}_{A\left(t_{0}\right)}\right]\left(t-t_{0}\right)
\end{gathered}
$$


Onde:

$\overrightarrow{\mathrm{X}}_{\mathrm{B}(\mathrm{t})}$ é o vetor com as coordenadas de um dado ponto $\mathrm{P}$ no sistema de referência $B$ na época $t$;

$\overrightarrow{\mathrm{T}}$ representa o vetor da translação necessária que faz com que os referenciais $\mathrm{A}$ e B coincidam;

s é o fator diferencial de escala entre os dois sistemas;

$\varepsilon$ é a matriz de rotação (em radianos) em torno do sistema cartesiano XYZ no referencial A que estabelece paralelismo com o referencial B na época $t_{0}$;

$\overrightarrow{\mathrm{V}}_{\mathrm{A}\left(\mathrm{t}_{0}\right)}$ representa o vetor das velocidades da estação a ser transformada. Tais velocidades, no presente trabalho foram retiradas do modelo VEMOS2009;

$\left(\mathrm{t}-\mathrm{t}_{0}\right)$ é o intervalo de tempo, em anos, decorrido entre a realização do sistema de referência $A\left(t_{0}\right)$ e a época do rastreio $(t)$;

$\dot{\overrightarrow{\mathrm{T}}}$, $\dot{\varepsilon}$ e $\dot{\mathrm{S}}$ são as variações temporais da translação, rotação e escala respectivamente.

Os dados processados estarão referenciados ao ITRFyy aplicado na TGH (e.g. ITRF05). Para se utilizar as coordenadas nos cálculos dos deslocamentos, é necessária uma nova aplicação da equação (1), já que a comparação deve ser realizada em uma mesma época e em um mesmo referencial (e.g. ITRF00, compatível com o SIRGAS2000). Os parâmetros de transformação entre os diferentes ITRFyy estão disponíveis na página do ITRF (http://itrf.ensg.ign.fr/ trans_para.php).

\subsection{Processamento dos dados}

O processamento dos dados GPS foi realizado no aplicativo comercial Leica Geo Office v.7.0. A primeira etapa do processamento constitui-se em determinar as coordenadas do pilar P1, tomado como ponto de referência da rede geodésica da UHE Salto Caxias, em relação a três estações de monitoramento contínuo pertencentes à Rede Brasileira de Monitoramento Contínuo - RBMC (PRMA Maringá/PR, PRGU - Guarapuava/PR e SCCH - Chapecó/SC) além da base localizada na barragem de Itaipu Binacional (ITAI - Foz do Iguaçu/PR).

A saber, as estações PRGU, PRMA, SCCH e ITAI distam 202 km, 284 km, $197 \mathrm{~km}$ e $110 \mathrm{~km}$ respectivamente da UHE Salto Caxias.

As coordenadas oficiais de tais estações, referenciadas ao SIRGAS2000 (ITRF00) foram transformadas e atualizadas para o ITRF05 ou ITRF08 (dependente da época de rastreio) e então o processamento foi realizado, fornecendo assim, coordenadas do $\mathrm{P} 1$ nos referenciais relacionados às efemérides precisas.

Toma-se como nota que o pilar P1 foi adotado como origem da rede geodésica em questão pelo fato de o mesmo estar em um local considerado estável (sobre formação rochosa) e ter baixa interferência na recepção dos sinais GPS.

O processamento foi realizado com os dados RINEX disponibilizados gratuitamente pelo IBGE após a aplicação da TGH nas coordenadas oficiais das mesmas, referenciando-se assim, o resultado ao ITRF05 ou ITRF08 (dependente da 
época de rastreio). Conforme já explicitado, a aplicação da TGH torna-se necessária devido à incompatibilidade entre as diferentes realizações do ITRS.

Com as coordenadas do pilar P1 determinadas, realiza-se então a segunda etapa do processamento, em que as coordenadas dos outros pilares (P3, P4, P5 e P6) e pino na crista da barragem $(\mathrm{C} 14)$ são determinadas em relação ao $\mathrm{P} 1$.

No processamento, alguns parâmetros foram adotados, a saber:

- Solução: Fixa;

- Frequência: L1/L2, e;

- Observável: Fase da portadora.

\subsection{Transformação de coordenadas - Sistema Geocêntrico para Sistema Geodésico Local}

Para a devida avaliação e interpretação dos deslocamentos, as coordenadas, que até então estavam referenciadas a um sistema geocêntrico serão transformadas para um sistema geodésico local. A partir do conjunto de equações a seguir, apresentados em Rodrigues (2002) e Jekeli (2006) é possível se realizar essa transformação.

$$
\begin{gathered}
{\left[\begin{array}{l}
e^{e} \\
n \\
u
\end{array}\right]=\left[R_{1}\left(90^{\circ}-\varphi_{0}\right) R_{3}\left(90^{\circ}+\lambda_{0}\right)\right]\left[\begin{array}{c}
X_{P}-X_{0} \\
Y_{P}-Y_{0} \\
Z_{P}-Z_{0}
\end{array}\right]} \\
R_{1}(\alpha)=\left[\begin{array}{ccc}
1 & 0 & 0 \\
0 & \cos (\alpha) & \operatorname{sen}(\alpha) \\
0 & -\operatorname{sen}(\alpha) & \cos (\alpha)
\end{array}\right] \\
R_{3}(\alpha)=\left[\begin{array}{ccc}
\cos (\alpha) & \operatorname{sen}(\alpha) & 0 \\
-\operatorname{sen}(\alpha) & \cos (\alpha) & 0 \\
0 & 0 & 1
\end{array}\right]
\end{gathered}
$$

Onde:

$(e, n, u)$ representa o terno de coordenadas topocêntricas do ponto a ser transformado;

$\mathrm{R}_{1}$ e $\mathrm{R}_{3}$ são matrizes de rotação em torno dos eixos $\mathrm{X}$ e $\mathrm{Z}$ respectivamente;

$\left(\varphi_{0}, \lambda_{0}\right)$ são as coordenadas geodésicas do ponto tomado como origem do Sistema Geodésico Local;

$\left(\mathrm{X}_{0}, \mathrm{Y}_{0}, \mathrm{Z}_{0}\right)$ são as coordenadas cartesianas do ponto tomado como origem do Sistema Geodésico Local, e;

$\left(\mathrm{X}_{\mathrm{P}}, \mathrm{Y}_{\mathrm{P}}, \mathrm{Z}_{\mathrm{P}}\right)$ são as coordenadas cartesianas geocêntricas do ponto a ser transformado do Sistema Geocêntrico para o Geodésico Local.

\subsection{Validação dos deslocamentos}

A validação dos deslocamentos foi realizada a partir do Teste de Congruência Global (TCG). Tal teste é baseado no Teste F de Fisher-Snedecor e identifica se um 
ponto se deslocou entre duas diferentes épocas. No TCG é preconizado pelo seguinte teste de hipóteses (CASPARY, 2000):

$$
\begin{gathered}
H_{0}: E\{d\}=E\left\{X_{A i}-X_{A j}\right\}=0 \\
H_{1}: E\{d\} \neq 0
\end{gathered}
$$

Com $H_{0}$ representando a hipótese básica, onde não ocorre o deslocamento $(d)$ dos pontos que compõem a rede e $H_{1}$, por sua vez, representando a hipótese alternativa, onde há deslocamento dos pontos.

$\mathrm{O}$ teste de hipótese testa os seguintes vetores:

$$
\left\{X_{A i}-X_{A j}\right\}=\left[\begin{array}{c}
X_{A i}-X_{A j} \\
Y_{A i}-Y_{A j} \\
Z_{A i}-Z_{A j}
\end{array}\right]
$$

Onde:

$X_{A i}$ e $X_{A j}$ representam os vetores das coordenadas do ponto A nas épocas i e j;

As matrizes variância-covariância (MVC) das coordenadas do ponto A em uma dada época (i,j) são conhecidas do ajustamento e são dadas por: $\Sigma X_{A i}$ e $\Sigma X_{A j}$. MVC.

Sendo possível então obter o deslocamento entre os pilares e sua respectiva

$$
\begin{gathered}
d=X_{A i}-X_{A j} \\
\Sigma d=\Sigma X_{A i}+\Sigma X_{A j}
\end{gathered}
$$

As covariâncias dos deslocamentos entre as diferentes épocas são dadas por:

$$
\sigma X_{A(i, j)}^{2}=\frac{r_{i} \sigma X_{A i}^{2}+r_{j} \sigma X_{A j}^{2}}{r_{i j}}
$$

Onde: $\mathrm{j}$;

$\sigma X_{A(i, j)}^{2}$ representa as covariâncias das coordenadas do ponto A nas épocas i e $\sigma X_{A i}^{2}$ e $\sigma X_{A j}^{2}$ são as variâncias das coordenadas do ponto $\mathrm{A}$, nas épocas i e j respectivamente;

$r_{i}$ e $r_{j}$ são os graus de liberdade de cada época, e;

$r_{i j}$ é o grau de liberdade da solução geral, dado por:

$$
r_{i j}=r_{i}+r_{j}
$$


Por fim, o TCG é aplicado:

$$
F_{C}=\frac{\left(\frac{\Omega}{h}\right)}{\sigma X_{A(i, j)}^{2}}
$$

Onde:

$h$ é a característica da matriz $\Sigma d$, e;

$$
\Omega=d^{T} \Sigma d^{-1} d
$$

\section{RESULTADOS}

\subsection{Deslocamentos calculados}

Após o cálculo das coordenadas geodésicas locais, é possível comparar os dados obtidos entre as campanhas. A campanha de Fevereiro/2009 foi tomada como época de referência, já que é a primeira época com dados GPS de todos os pontos de interesse. Na tabela 3 são representadas as diferenças entre as coordenadas geodésicas locais obtidas em Fevereiro/2009 e as demais campanhas.

Tabela 3 - Diferenças, em milímetros, entre as coordenadas geodésicas locais

\begin{tabular}{|c|c|c|c|c|c|c|c|c|c|c|c|c|}
\hline CAMPANHA & \multicolumn{3}{|c|}{ Junho/2010 } & \multicolumn{3}{|c|}{ Outubro/2010 } & \multicolumn{3}{|c|}{ Maio/2011 } & \multicolumn{3}{|c|}{ Outubro/2011 } \\
\hline$\frac{\text { COORDENADA }}{\text { PILAR }}$ & $\Delta e$ & $\Delta n$ & $\Delta u$ & $\Delta e$ & $\Delta n$ & $\Delta u$ & $\Delta e$ & $\Delta n$ & $\Delta u$ & $\Delta e$ & $\Delta n$ & $\Delta u$ \\
\hline P6 & $-0,5$ & 0,6 & 22,9 & $-1,9$ & $-22,1$ & 9,4 & $-0,8$ & $-2,1$ & 0,9 & 0,9 & 1,0 & 3,1 \\
\hline $\mathrm{C} 14$ & $-1,4$ & $-5,0$ & $-4,8$ & $-1,7$ & $-25,0$ & 2,7 & $-0,8$ & $-6,2$ & $-6,1$ & 3,9 & $-3,1$ & 3,4 \\
\hline P5 & 0,0 & $-2,2$ & $-1,0$ & $-3,4$ & $-23,4$ & $-3,7$ & $-3,7$ & $-6,2$ & $-12,7$ & $-8,1$ & $-2,5$ & 0,7 \\
\hline $\mathrm{P} 4$ & 0,5 & $-0,7$ & $-1,3$ & $-0,6$ & $-22,5$ & 8,6 & 1,4 & $-1,9$ & $-6,5$ & 3,6 & 6,4 & $-1,0$ \\
\hline P3 & 0,8 & 0,3 & $-1,7$ & $-1,0$ & $-22,5$ & 9,7 & 0,8 & $-1,5$ & $-14,6$ & 5,0 & 0,6 & 4,3 \\
\hline
\end{tabular}
obtidas em Fev/2009 (época de referência) e as demais campanhas.

As Figuras a seguir ilustram os deslocamentos apresentados na Tabela 3. 
Figura 2 - Variação das coordenadas do pilar P6.

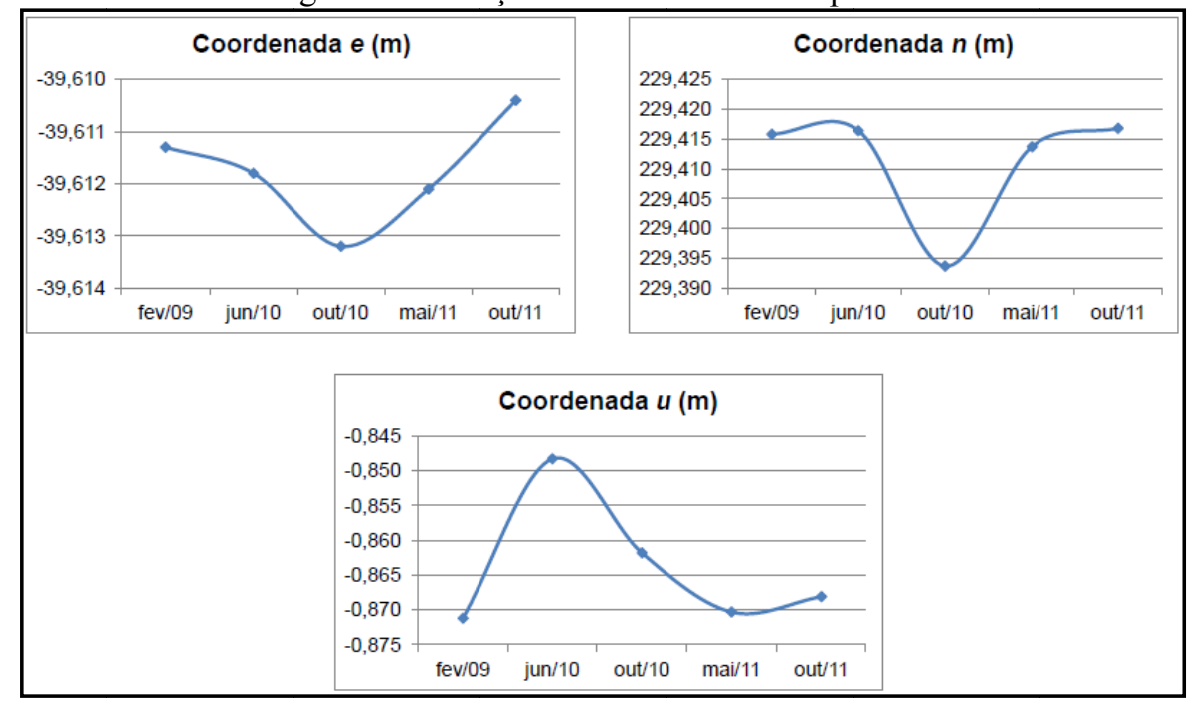

Figura 3 - Variação das coordenadas do ponto C14.

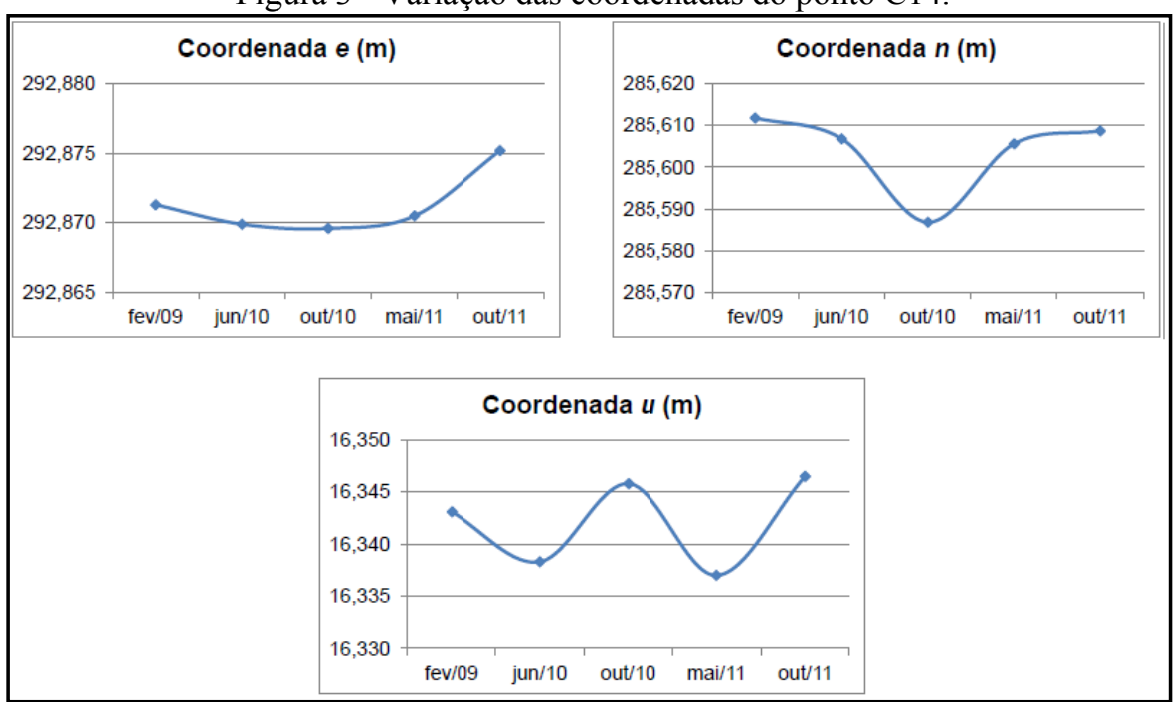

Bol. Ciênc. Geod., sec. Artigos, Curitiba, v. 18, nº 4, p.564-582, out-dez, 2012. 
Figura 4 - Variação das coordenadas do pilar P5

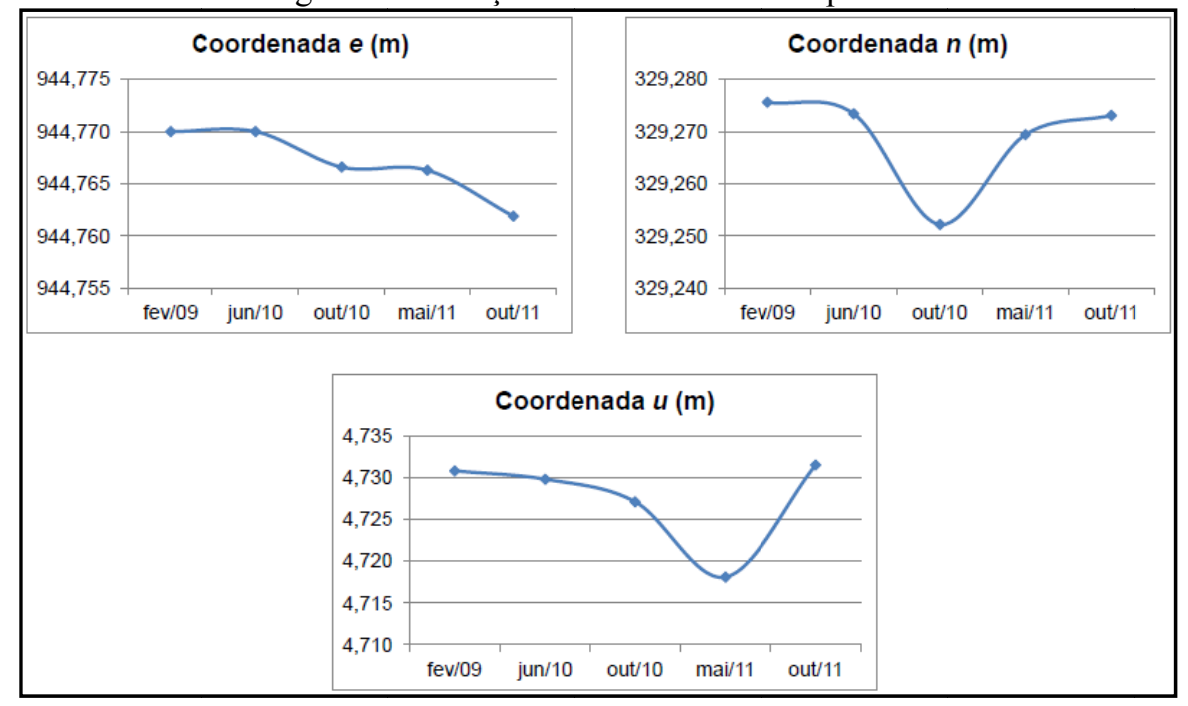

Figura 5 - Variação das coordenadas do pilar P4.

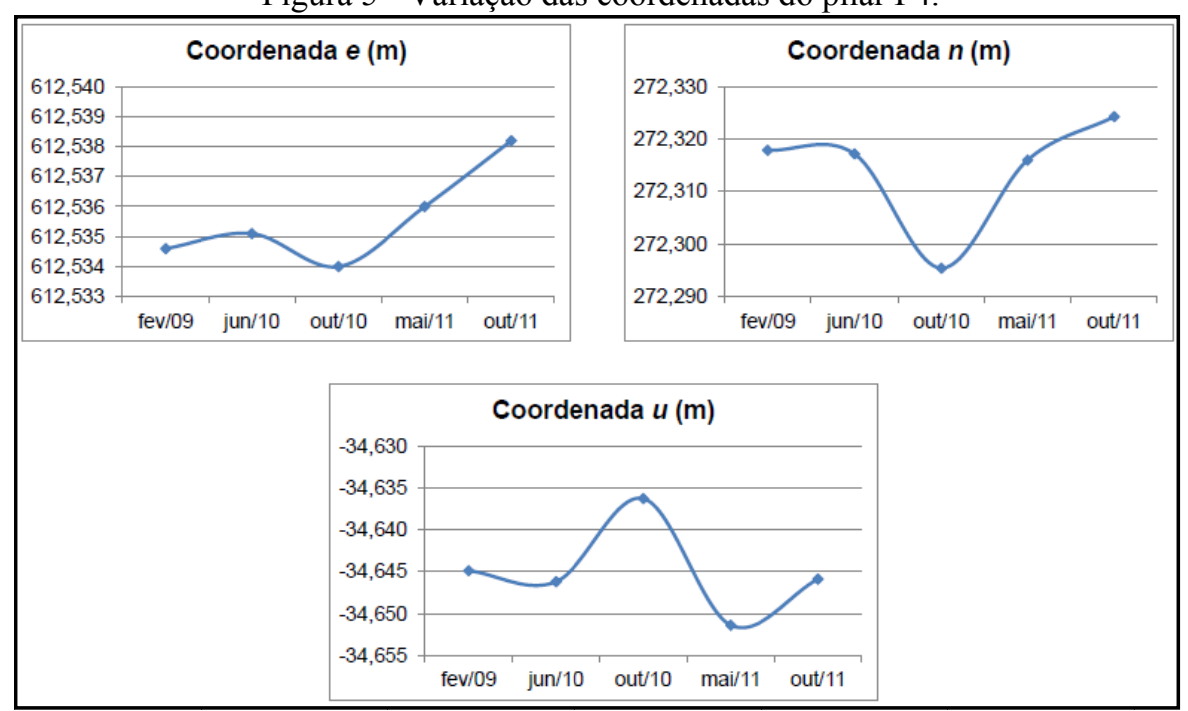

Bol. Ciênc. Geod., sec. Artigos, Curitiba, v. 18, no 4, p.564-582, out-dez, 2012. 
Figura 6 - Variação das coordenadas do pilar P3.

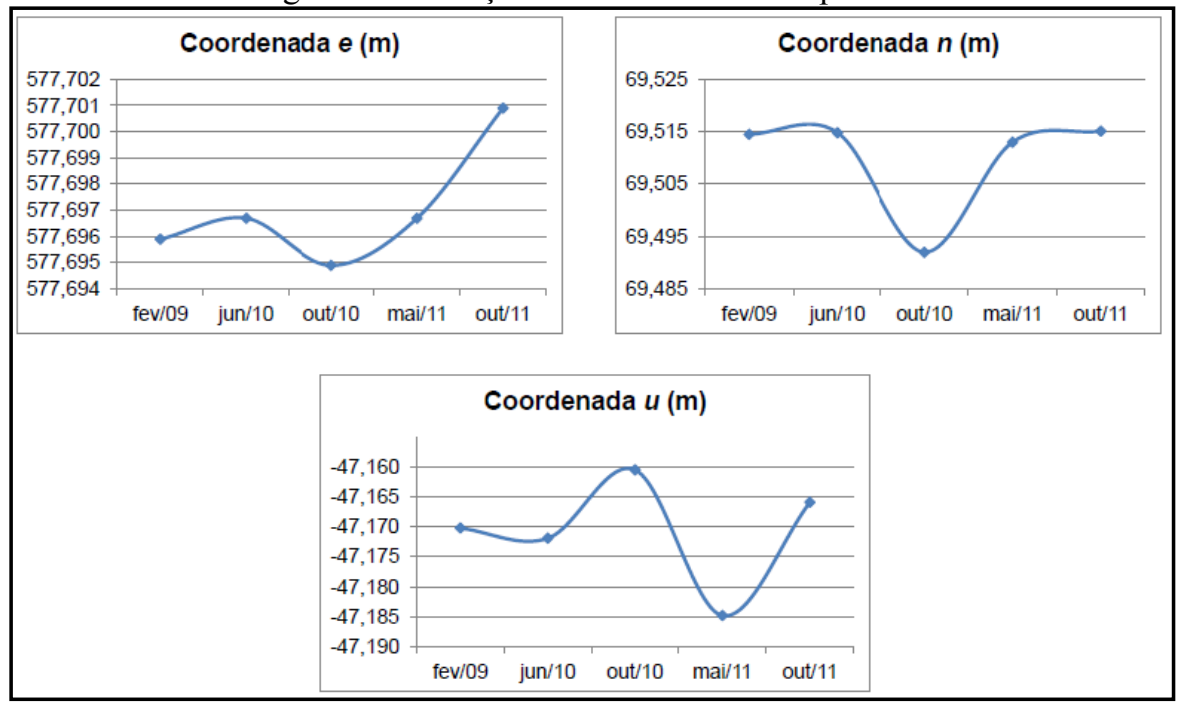

\subsection{Validação dos deslocamentos - TCG}

Para que os deslocamentos sejam validados, foi aplicado o TCG (3.6). Já que as épocas analisadas possuem o mesmo grau de liberdade, o valor de $\mathrm{F}$ tabelado foi 2,7758 (com 95\% de grau de confiança, $\alpha=0,05$ ). Então, caso o valor de $\mathrm{F}$ calculado seja maior que o tabelado, é sinal de que ocorreu o deslocamento.

Nas tabelas a seguir $(4,5,6$ e 7) são apresentados os resultados da aplicação dos testes nos deslocamentos calculados entre a época de referência e as demais.

As células destacadas nas tabelas indicam em qual componente da coordenadas do pilar que houve deslocamento $\left(F_{C A L C}>F_{T A B}\right)$.

Estatisticamente, além da componente $n$ do pilar P6 (Jun/10 - Tabela 4), todas as componentes das coordenadas de todos os pilares da época Out/10 (Tabela 5) foram considerados deslocamentos. Tal resultado sugere que pode haver um erro sistemático nos dados de toda a campanha, já que erros grosseiros não foram encontrados no Teste Data Snooping.

Ressalta-se que as coordenadas analisadas foram atualizadas para uma mesma época (Fev/09) e no mesmo sistema de referência (SIRGAS2000, compatível com o ITRF00). Essa atualização/transformação foi realizada a partir da TGH (3.3). 
Tabela 4 - Valores de F calculados para os deslocamentos entre a época de referência (fev/09) e jun/10.

\begin{tabular}{c|c|c|c}
\hline PILAR & $e$ & $n$ & $u$ \\
\hline P6 & 0,9736 & 2,9208 & 1,1248 \\
\hline C14 & 0,2805 & 0,5610 & 0,1870 \\
\hline P5 & 0,0287 & 0,0862 & 0,5390 \\
\hline P4 & 0,0063 & 0,0189 & 0,0094 \\
\hline P3 & 0,0070 & 0,0211 & 0,0028 \\
\hline
\end{tabular}

Tabela 5 - Valores de F calculados para os deslocamentos entre a época de referência (fev/09) e out/10.

\begin{tabular}{c|c|c|c}
\multicolumn{4}{|c}{ referencia (fev/09) e out/10. } \\
\hline PILAR & $e$ & $n$ & $u$ \\
\hline P6 & 2,8837 & 8,6511 & 3,1128 \\
\hline C14 & 5,2406 & 10,4813 & 3,4938 \\
\hline P5 & 3,0888 & 9,2663 & 5,4934 \\
\hline P4 & 2,9501 & 8,8504 & 4,4133 \\
\hline P3 & 2,9886 & 8,9658 & 2,9848 \\
\hline
\end{tabular}

Tabela 6 - Valores de F calculados para os deslocamentos entre a época de referência (fev/09) e mai/11.

\begin{tabular}{c|c|c|c}
\hline PILAR & $e$ & $n$ & $u$ \\
\hline P6 & 0,0272 & 0,0816 & 0,0359 \\
\hline C14 & 0,4264 & 0,8527 & 0,2842 \\
\hline P5 & 0,5376 & 1,6128 & 0,8872 \\
\hline P4 & 0,1019 & 0,3058 & 0,2033 \\
\hline P3 & 0,4084 & 1,2253 & 0,7365 \\
\hline
\end{tabular}

Tabela 7 - Valores de F calculados para os deslocamentos entre a época de referência (fev/09) e out/11.

\begin{tabular}{c|c|c|c}
\hline PILAR & $e$ & $n$ & $u$ \\
\hline P6 & 0,0249 & 0,0746 & 0,0560 \\
\hline C14 & 0,1756 & 0,3511 & 0,1170 \\
\hline P5 & 0,1571 & 0,4714 & 0,5048 \\
\hline P4 & 0,2534 & 0,7602 & 0,4688 \\
\hline P3 & 0,0825 & 0,2476 & 0,1004 \\
\hline
\end{tabular}




\subsection{Comparação - LPS e GPS}

As distâncias entre os pilares obtidas a partir dos dados GPS foram comparadas com essas mesmas distâncias obtidas utilizando a Estação Total Leica TC2003 com precisão linear de $\pm(1 \mathrm{~mm}+1 \mathrm{ppm})$ e angular de $\pm 0,5$ ".

As distâncias obtidas pela TC2003 estão referenciadas a um sistema local e corrigidas dos efeitos atmosféricos (pressão, umidade e temperatura). Já as distâncias obtidas a partir dos dados GPS estão referenciadas a um sistema geodésico local (3.5). No presente trabalho, pela pequena extensão da rede, os dois sistemas mencionados foram adotados como coincidentes, com a finalidade de viabilizar a comparação entre as técnicas.

$\mathrm{Na}$ tabela a seguir, serão apresentadas as diferenças entre as distâncias obtidas com as duas técnicas nas cinco épocas analisadas (Fev/09, Jun/10, Out/10, Mai/11 e Out/11).

Tabela 5 - Diferenças (em mm) das distâncias entre pilares, obtidas a partir de dados GPS e dados obtidos com a Estação Total Leica TC2003.

\begin{tabular}{c|c|c|c|c|c}
\hline PILARES & Fev/09 & Jun/10 & Out/10 & Mai/11 & Out/11 \\
\hline P1 - P6 & $-6,0$ & $-4,5$ & 17,8 & $-2,1$ & $-4,9$ \\
\hline P1 - C14 & $-12,6$ & $-7,9$ & 6,5 & $-6,3$ & $-13,7$ \\
\hline P1 - P5 & $-26,2$ & $-21,1$ & $-10,5$ & $-13,8$ & $-14,2$ \\
\hline P1 - P4 & $-11,9$ & $-7,8$ & 3,6 & $-7,3$ & $-14,8$ \\
\hline P1 - P3 & $-14,8$ & $-5,4$ & 79,6 & $-1,8$ & $-9,0$ \\
\hline P6 - C14 & - & - & - & - & - \\
\hline P6 - P5 & $-24,8$ & $-20,6$ & $-19,1$ & $-16,9$ & $-13,4$ \\
\hline P6 - P4 & $-11,3$ & $-9,4$ & $-8,6$ & $-10,1$ & $-12,7$ \\
\hline P6 - P3 & $-15,1$ & $-9,6$ & 64,8 & $-7,9$ & $-11,0$ \\
\hline C14 - P5 & - & - & - & - & - \\
\hline C14 - P4 & - & - & - & - & - \\
\hline C14 - P3 & $-8,1$ & 1,2 & 175,9 & 0,3 & 2,1 \\
\hline P5 - P4 & $-4,6$ & $-1,9$ & $-0,2$ & 3,0 & 9,6 \\
\hline P5 - P3 & - & - & - & - & - \\
\hline P4 - P3 & $-4,8$ & 0,9 & 58,6 & 0,0 & $-5,6$ \\
\hline
\end{tabular}

Nota-se os valores máximo de 26,2 mm (Fev/09, P1-P5) e mínimo de 0,0 (Mai/11, P4-P3), com exceção da campanha de Out/10, que conta com valores bastante discrepantes das demais épocas (79,6 mm, P1-P3; 64,8 mm, P6-P3; 175,9 $\mathrm{mm}, \mathrm{C} 14-\mathrm{P} 3 \mathrm{e} ; 58,6 \mathrm{~mm}, \mathrm{P} 4-\mathrm{P} 3$ ), o que remete ao que já foi discutido no tópico 4.2 .

Por conta das altas discrepâncias que ocorreram na campanha de Out/10 (Tabela 5), optou-se por suprimi-la da Figura 6, melhorando a visualização das discrepâncias das outras campanhas.

As distâncias (P6-C14; C14-P5; C14-P4 e; P5-P3) não foram comparadas pois em campo as visadas entre os pares de pilares não são factíveis. 
Fig. 6 - Diferenças entre as distâncias, entre cada par de pilares, obtidas com a Estação Total Leica TC2003 e com dados GPS (com exceção da campanha Out/10).

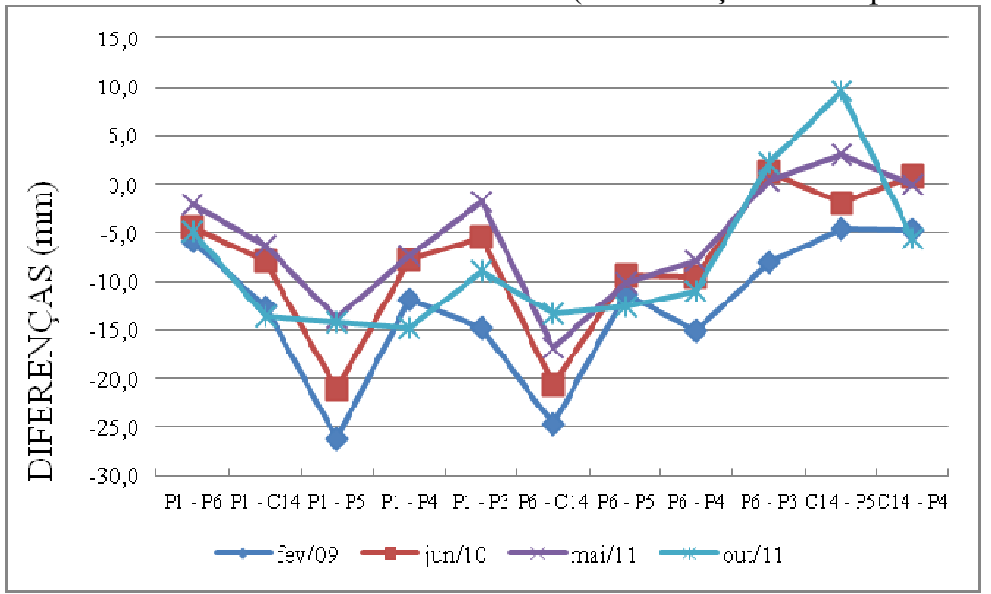

Propagou-se a variância para o cálculo da distância entre os pilares a partir das coordenadas no sistema geodésico local (e, n, u). Os resultados obtidos, apresentados na Tabela 5 a seguir demonstram que a maioria (60\%) das discrepâncias alcançadas (Tabela 6) está acima das precisões calculadas pela propagação.

Tabela 6 - Precisões calculadas para as distâncias obtidas com os dados

\begin{tabular}{c|c|c|c}
\hline \multicolumn{2}{c|}{ PONTOS } & DISTÂNCIA & $\begin{array}{c}\text { PRECISÃO } \\
\text { NOMINAL }(\mathrm{mm})\end{array}$ \\
\hline P1 & P6 & 232,806 & 5,98 \\
\hline P1 & C14 & 409,395 & 6,04 \\
\hline P1 & P5 & 1000,491 & 7,69 \\
\hline P1 & P4 & 671,223 & 7,49 \\
\hline P1 & P3 & 583,757 & 7,53 \\
\hline P6 & C14 & - & - \\
\hline P6 & P5 & 989,424 & 6,50 \\
\hline P6 & P4 & 654,416 & 6,29 \\
\hline P6 & P3 & 639,344 & 6,25 \\
\hline C14 & P5 & - & - \\
\hline C14 & P4 & - & - \\
\hline C14 & P3 & 363,113 & 6,13 \\
\hline P5 & P4 & 339,370 & 6,99 \\
\hline P5 & P3 & & - \\
\hline P4 & P3 & 206,150 & 6,97 \\
\hline
\end{tabular}




\section{CONCLUSÕES}

De acordo com os resultados obtidos, foi possível identificar os deslocamentos ocorridos nos pilares (P3, P4, P5 e P6) e no pino (C14) que compõem a rede geodésica que dá suporte ao monitoramento da UHE Salto Caxias.

As Figuras 2, 3, 4, 5 e 6 ilustram o comportamento aleatório desses deslocamentos, o que foi de encontro às expectativas dos autores, já que o monitoramento dos pilares realizado com outra técnica (triangulateração com a Estação Total Leica TC 2003) não detectou nenhum deslocamento relativo entre os mesmos.

O pilar P1 não foi analisado, pois o mesmo foi tomado como origem da rede geodésica local e de acordo com a Eq. (2), as coordenadas de tal pilar são iguais a $(0,0,0)$. Ressalta-se que no processamento, apesar de o pilar P1 ter sido injuncionado e tomado como origem da rede, a incerteza inerente a ele foi levada em consideração no processamento das coordenadas dos demais pilares. Tal incerteza é originária da determinação das coordenadas de tal pilar em relação às estações de monitoramento contínuo utilizadas como base no primeiro processamento, que por sua vez também tiveram suas variâncias consideradas no processamento dos dados GNSS.

A campanha de Out/10, conforme já explicado, pode estar contaminada de erros sistemáticos, já que erros grosseiros não foram identificados e os seus resultados ficaram bastante discrepantes das outras épocas (fato comprovado estatisticamente com o TCG).

Quanto à comparação das distâncias obtidas com GPS e Estação Total, concluiu-se que foi possível identificar diferenças que variaram de $0,0 \mathrm{~mm}$ a 26,2 $\mathrm{mm}$, ficando acima das precisões nominais dos aparelhos utilizados (Tabela 2), com os maiores erros relacionados às duas maiores distâncias (P1-P5 e P6-P5), com médias de discrepâncias iguais a 18,8 e 18,9 mm respectivamente, ressalta-se que o pilar P5 está instalado em um local vulnerável ao efeito de multicaminhamento, o que pode ter causado a degradação dos resultados relacionados a esse pilar.

Maiores estudos devem ser realizados em relação à rede geodésica da UHE Salto Caxias para avaliar possíveis influências de condições locais (obstruções, linhas de transmissão e condições atmosféricas) sobre a qualidade dos dados GPS.

\section{AGRADECIMENTOS}

Esta pesquisa teve o incentivo da Universidade Federal do Paraná através do Setor de Ciências da Terra, Departamento de Geomática e Curso de Pós-Graduação em Ciências Geodésicas que colocaram à disposição os equipamentos, instalações e aplicativos computacionais do Laboratório de Topografia (LABTOPO) e de Instrumentação e Aferição Geodésica (LAIG).

$\mathrm{O}$ apoio financeiro foi dado pelo CNPq através da concessão da bolsa de estudos durante o período de mestrado do primeiro autor. 
Este trabalho encontra-se vinculado ao projeto "Integração da Auscultação Geodésica com a Instrumentação de controle e Segurança de Barragens", sob o registro BANPESQ: 200.301.308-8.

\section{REFERÊNCIAS BIBLIOGRÁFICAS}

CASPARY, W.F. Concepts of network and deformation analysis. Kensigton, N.S.W., Australia: School of Geomatic Engineering, The University of New South Wales, 2000. 183 p.

COPEL - Companhia Paranaense de Energia. Geração. 2008. Disponível em: $<$ http://www.copel.com/hpcopel/root/nivel2.jsp?endereco=\%2Fhpcopel $\% 2$ Froot\%2Fpagcopel2.nsf\%2F044b34faa7cc1143032570bd0059aa29\%2F9bdc3 7f6b8c44b810325741200587db7>. Acessado em 7 de Abril de 2011.

DREWES H., HEIDBACH O. The 2009 horizontal velocity model for South America and the Caribbean. Submitted to C. Pacino et al. (Eds.). IAG Scientific Assembly "Geodesy for Planet Earth". Buenos Aires, August 31 to September 4, 2009. IAG Symposia.

DUFFY, M.A, et.al. An automated and integrated monitoring program for Diamond Valley Lake in California. In: $10^{\text {th }}$ FIG Intl. Symp. On Deformation Measurements, Orange, March 19 to March 22, 2001.

FAZAN, J.A. Aplicação do teste de congruência global e análise geométrica para detecção de deslocamentos em redes geodésicas: Estudo de caso na UHE de Itaipu, Dissertação (Mestrado) - Escola Politécnica da Universidade de São Paulo, São Paulo, 2010.

GRANEMANN, D.C. Estabelecimento de uma rede geodésica para o monitoramento de estruturas: estudo de caso na Usina Hidrelétrica Salto Caxias, Dissertação (Mestrado) - Curso de Pós Graduação em Ciências Geodésicas, Departamento de Geomática, Universidade Federal do Paraná, Curitiba, PR, 2005.

JUNIOR, B.M., SOARES, M.A., A Auscultação dos Instrumentos da Barragem de Salto Caxias. In: Simpósio sobre instrumentação de barragens, 3., 2006, São Paulo. Anais. p. 35 - 46.

KÄLBER, S.; JÄGER, R; SCHWÄBLE, R.: HEIMBERG, F. and K. KAST (2000): GPS-based Online Control and Alarm System (GOCA). Report presented to the 20. ICOLD-Congress, 2000, Peking. International Commission on Large Dams, Paris.

JEKELI, C. Geometric Reference Systems in Geodesy. Ohio State University, 202p. 2006. Lecture Note.

LEICK, A. GPS Satellite Surveying. 3. ed. Hoboken; New Jersey: John Wiley e Sons, 2004.

MONICO, J.F.G. Posicionamento pelo GNSS: Descrição, fundamentos e aplicações. São Paulo: Editora Unesp, 476 p., 2008.

NADAL, M.A.D. Controle e Operação remota de Estações Totais Robotizadas voltado à Auscultação Geodésica, Dissertação (Mestrado) - Curso de Pós- 
Graduação em Ciências Geodésicas, Departamento de Geomática, Universidade Federal do Paraná, Curitiba, PR, 2008.

NEVES, R.V. Processamento e Análise de levantamentos geodésicos aplicado ao monitoramento de estruturas civis, Dissertação (Mestrado) - Curso de PósGraduação em Ciências Geodésicas, Departamento de Geomática, Universidade Federal do Paraná, Curitiba, PR, 2008.

RIBEIRO, F.C.D. Proposta de metodologia para verificação da estabilidade dos pilares de redes para monitoramento geodésico de barragens - estudo de caso. Dissertação (Mestrado) - Escola Politécnica da Universidade de São Paulo, São Paulo, 2008.

RODRIGUES, D.D. Rede geodésica de precisão no Estado de Minas Gerais: avaliação de diferentes estratégias de processamento e ajustamento. Tese (Doutorado) - Escola Politécnica - Universidade de São Paulo, São Paulo, 2002.

SEEBER, G. Satellite geodesy: foundations, methods and applications. Berlin; New York: de Gruyter, 1993. 356p.,

US ARMY, Structural Deformation Surveying. Washington: Engineer Manual, 2002. 292p.

USINA Hidrelétrica Salto Caxias. Planta geral; instrumentação existente; arranjo geral, planta e perfil. 2003. 1 CD ROM. 1:2000.

(Recebido em de março de 2012. Aceito em outubro de 2012.) 